\title{
Définition de l'aléa et des risques dans les projets d'infrastructures en zone karstique
}

\section{J. MANIA}

Laboratoire de mécanique de Lille (CNRS, UMR 8107)

Cité scientifique

59655 Villeneuve-d'Ascq

Cedex

<jacky.mania@eudil.fr>
La présence des anomalies de sub-surface liées à la dissolution des formations calcaires par l'activité corrosive des eaux superficielles entraîne des risques d'instabilité mécanique et de faisabilité à un coût optimal des ouvrages et des fondations. Lors de la réalisation de grandes infrastructures en vue de la construction des voies de circulation routière ou ferrée il est souvent délicat d'implanter des sondages de reconnaissance sans une évaluation des risques d'apparition de désordres souterrains. Une méthodologie probabiliste est développée afin de conduire à une meilleure estimation des aléas en intégrant les observations de terrain. Un exemple d'analyse statistique portant sur $173,5 \mathrm{~km}$ de linéaire en zone karstique de I'Est de la France permet de conforter l'extrapolation de la cartographie synthétique et l'investigation complémentaire géotechnique. Nous utilisons la loi des événements rares de la loi de Poisson pour calculer par le test du $\mathrm{CHI}^{2}$ la normalité de la distribution des phénomènes karstiques. Enfin nous présentons des applications de la méthodologie avec une amélioration du calcul du coefficient de risque $\mathrm{R}$ et de l'index de karstification Ik.

Mots-clés : infrastructures routières, infrastructures ferrées, karst, instabilité, effondrement.

\section{Hazards assessment for infrastructure project in karstic are}

The dissolution of limestone formations by the corrosive activity of superficial waters leads to important risk of mechanical instability, which has to be considered in infrastructure project. Geotechnical investigation for infrastructure project requires assessment of the risks related to subsurface disorders. A probabilistic methodology is proposed in order to improve hazards assessment by using in situ observations. An example of a statistical analysis concerning $173.5 \mathrm{~km}$ of shelf space in karstic zone of eastern part of France allows consolidating the extrapolation of the synthetic cartography and the additional geotechnical investigation. The law of the rare events of Poisson is used to calculate by the test of the $\mathrm{CHI}^{2}$ the normality of the distribution of the karstic features, Finally we present applications of the methodology with an improvement of the calculation of the risk coefficient $\mathrm{A}$ and of the karstification index Ik.

Key words : railroad infrastructures, railway infrastructures, karstic areas, instability, collapse. 


\section{Introduction}

Les terrains carbonatés constituent en raison des vides créés par le phénomène de karstification des secteurs préjudiciables aux tracés d'infrastructures linéaires tels que les nouvelles voies ferrées ou les autoroutes. Outre l'effondrement des voûtes minces ou broyées, les zones karstiques peuvent subir une évolution géomorphologique actuelle, éventuellement réactivée par une modification de l'écoulement des eaux apportée par le tracé lui-même (reprise d'érosion). Dans notre cas compte tenu de la spécificité géomorphologique et géotechnique du milieu, une méthodologie adaptée à ce milieu particulier a été définie. Son objectif final est d'établir une cartographie des facteurs de risque fondée sur un recensement d'indices de terrain hiérarchisés pour l'élaboration d'une grille de critères dont la pertinence a été testée (Mania et al., 1998).

La cartographie géotechnique (Sanejouand, 1972) introduit l'analyse de différents facteurs géotechniques. L'analyse des risques naturels liés aux mouvements de terrain est très courante et aboutit à la réalisation d'une carte des degrés du risque d'instabilité basée sur plusieurs paramètres parmi lesquels la nature lithologique et géotechnique des formations géologiques (Fares et al., 1994). Dans le domaine qualitatif, l'examen de la vulnérabilité du milieu karstique a fait l'objet de nombreux travaux en particulier au niveau de la protection des eaux souterraines (Vrba et Zaporozec, 1994 ; Doerfliger et al., 1997 ; Tripet et al., 1997) dans un but cartographique et thématique intéressant les aménageurs.
Dans notre cas, la démarche est similaire, car nous désirons répondre aux répercussions des risques géotechniques sur les futurs linéaires du réseau ferré (par exemple celui du TGV Mulhouse-Dijon). Il est important d'étudier le réseau karstique et les circulations d'eau souterraine en dressant un inventaire détaillé et une cartographie de tous les éléments témoignant de l'existence d'une activité karstique (Tableau I). Dans une première phase a été élaborée une carte des facteurs de risque sur les vallées de l'Ognon, sur l'interfluve Ognon, vallée du Doubs et vallée de la Saône (Mania et al., 1998).

\section{2}

\section{Méthodologie}

La première étape de terrain du recensement des phénomènes karstiques (sources, dolines, grottes, pertes, gouffres) a été suivie d'une analyse multicritère appliquée aux indices de terrain permettant de définir des facteurs de karstification puis d'établir une grille plausible des aléas potentiels vis-à-vis de l'infrastructure projetée. Dans cet article, l'apport de l'outil des probabilités complète l'approche des risques, afin d'affiner la méthode précédemment développée (Mania et al., 1998).

Le développement méthodologie de la prise en compte des phénomènes aléatoires karstiques met l'accent sur l'utilisation de l'outil des probabilités qui sera ici la loi de Poisson. Cette dernière est une loi de probabilité discrète qui correspond à une loi des " événements rares ). La variable aléatoire discrète $r$ (pré-

TABLEAU Répartition des phénomènes (ou événements) observés sur le terrain et liés à la dissolution des carbonates. Distribution of the observed karstic features (or events) in the field and linked to the limestone dissolution.

\begin{tabular}{|c|c|c|c|c|c|c|c|c|}
\hline Planche $n^{\circ}$ & $\begin{array}{l}\text { Distances } \\
\text { en km }\end{array}$ & $\begin{array}{c}\text { Superficie } \\
\text { en } \mathrm{km}^{2}\end{array}$ & Sources & $\begin{array}{l}\text { Dolines } \\
\text { actives }\end{array}$ & $\begin{array}{l}\text { Dolines } \\
\text { colmatées }\end{array}$ & Grottes & $\begin{array}{c}\text { Pertes } \\
\text { de ruisseaux }\end{array}$ & Gouffires \\
\hline $\begin{array}{l}\text { PEO III } \\
\text { PEO IV } \\
\text { PEE I } \\
\text { PEE III } \\
\text { PEE II } \\
\text { PEE IV } \\
\text { GY III } \\
\text { GY II + IV } \\
\text { BEO I } \\
\text { BEO II } \\
\text { BEE I + II } \\
\text { VEE I + II } \\
\text { BAO I } \\
\text { BAO III } \\
\text { BAO II } \\
\text { BAE I } \\
\text { BAE II } \\
\text { LUO III } \\
\text { LUO IV } \\
\text { LUE III } \\
\text { LUE IV } \\
\text { BFO III } \\
\text { FLD I } \\
\text { FLD II } \\
\text { FLD III } \\
\text { FLD IV } \\
\text { MAR III } \\
\text { MAR IV + DIJ } \\
\text { AUX I }\end{array}$ & $\begin{array}{c}7 \\
7 \\
2 \\
6 \\
7 \\
2,5 \\
3 \\
12,5 \\
7 \\
7 \\
8 \\
4,5 \\
6 \\
1 \\
8 \\
7 \\
3,5 \\
7 \\
7 \\
7 \\
7 \\
1,5 \\
2,12 \\
5,8 \\
5,12 \\
7,25 \\
12,77 \\
5,3 \\
6,62\end{array}$ & $\begin{array}{c}23 \\
24 \\
3 \\
20 \\
14 \\
10 \\
6,2 \\
32,5 \\
22 \\
21 \\
23 \\
14 \\
18,1 \\
7,7 \\
24,8 \\
20 \\
3,5 \\
22,5 \\
22,5 \\
21 \\
23 \\
4,75 \\
6,02 \\
18,18 \\
13,78 \\
29,23 \\
28,91 \\
15,5 \\
16,96\end{array}$ & $\begin{array}{c}1 \\
2 \\
0 \\
13 \\
3 \\
4 \\
5 \\
27 \\
3 \\
4 \\
3 \\
1 \\
6 \\
2 \\
6 \\
8 \\
2 \\
12 \\
6 \\
0 \\
2 \\
0 \\
5 \\
3 \\
2 \\
0 \\
8 \\
10 \\
0\end{array}$ & $\begin{array}{c}1 \\
0 \\
0 \\
1 \\
5 \\
1 \\
0 \\
7 \\
11 \\
4 \\
10 \\
0 \\
1 \\
2 \\
34 \\
2 \\
0 \\
6 \\
3 \\
0 \\
15 \\
1 \\
4 \\
14 \\
13 \\
29 \\
17 \\
21 \\
0\end{array}$ & $\begin{array}{c}0 \\
2 \\
0 \\
1 \\
3 \\
8 \\
2 \\
3 \\
6 \\
2 \\
1 \\
0 \\
9 \\
3 \\
11 \\
7 \\
0 \\
0 \\
0 \\
9 \\
14 \\
0 \\
1 \\
3 \\
0 \\
3 \\
0 \\
0 \\
0\end{array}$ & $\begin{array}{l}0 \\
0 \\
0 \\
1 \\
1 \\
0 \\
0 \\
1 \\
0 \\
0 \\
1 \\
0 \\
0 \\
0 \\
0 \\
1 \\
0 \\
0 \\
1 \\
0 \\
0 \\
0 \\
1 \\
8 \\
15 \\
6 \\
0 \\
0 \\
0\end{array}$ & $\begin{array}{l}2 \\
1 \\
0 \\
4\end{array}$ & $\begin{array}{l}0 \\
0 \\
0\end{array}$ \\
\hline Total & 173,48 & 509,13 & 138 & 202 & 88 & 36 & 34 & 9 \\
\hline
\end{tabular}


sence d'une doline, d'un gouffre, d'une perte, etc.) qui correspond à des phénomènes observés par unité de distance ou d'espace (superficie du secteur d'investigation ou fuseau géographique du tableau I) peut suivre une loi de Poisson de paramètre a notée Pr si la fonction de probabilité est d'expression :

$$
\text { Prob. }(R=r)=\operatorname{Pr}=\frac{e^{-x} a^{x}}{r !}
$$

Si un grand nombre d'observations n sont faites alors $a=n P r, P r$ étant petit alors a est fini. Si sur les planches géographiques affectées par le tracé d'un linéaire la surface du fuseau est connue Stf et que $S$ j correspond à la surface d'une zone indicée $f$ les densités $\lambda=n / S t f$ et $\beta=n j / S j$, avec $n$ le nombre total d'événements de dissolution et nj le nombre d'événements spécifiques à une classe d'índice (Tableau II). La probabilité qu'un site géographique contienne une forme de dissolution sera définie par $\mathrm{Pr}=\lambda \mathrm{Sj} / \mathrm{nj}$. Si la largeur d'un fuseau géographique est petite par rapport à la longueur on peut substituer la surface Stf par la distance $D t f$ et $S j$ par $D j$. On obtient ainsi successivement les estimations de $\lambda=n / D t f, \beta=n j / D j$ et $P r=\lambda$ Dj/nj évalué dans le tableau II.
La comparaison des événements espérés statistiquement et réellement observés sur le terrain (Tableau III) est ensuite effectuée par l'intermédiaire du test classique du $\mathrm{CHI}^{2}$ (Tableau IV) suivant la relation classique :

$$
C H I^{2}=\sum_{j=1}^{r} \frac{(O j-P j)^{2}}{P j}
$$

oủ Oj est le nombre d'observations à l'intérieur de la je classe d'indice et $P j$ le nombre d'observations espérées calculées par la relation (1) dans cette classe.

On peut constater qu'en partant de quatre catégories statistiques $(r=4)$ avec ici deux degrés de liberté (un premier degré de liberté est affecté au fait que les fréquences espérées sont contraintes à une somme finie d'événements et un second degré de liberté est. nécessaire à l'estimation de $\lambda$ ) on aboutit à un test où la valeur critique du $\mathrm{CHI}^{2}$ pour un risque de $5 \%$ de nonréalisation est de 5,99. L'événement ( sources ) dépasse largement cette valeur et donc le test de Poisson n'est pas valable pour lui, car la répartition géographique n'est pas aléatoire (elle dépend en effet des contacts géologiques entre niveaux imperméables et niveaux perméables). Cet événement est ainsi abandonné dans le calcul des risques.

TABLEAUII Probabilités théoriques de répartition des événements observés sur le terrain.

Remarques: le paramètre de Poisson est évalué par rapport à la distance de $173,48 \mathrm{~km}$.

La variable discrète $\mathrm{r}$ limitée à 6 unités sera ensuite réduite à 5 .

Theoretical probabilities of the observed events distribution in the field.

Remarks: the Poisson law parameter is evaluated on a distance of $173.48 \mathrm{~km}$.

The discrete variable $r$ is limited to 6 units and then reduced to 5 units.

\begin{tabular}{l|c|c|c|c|c|c}
\hline Événement & Sources & $\begin{array}{c}\text { Dolines } \\
\text { actives }\end{array}$ & $\begin{array}{c}\text { Dolines } \\
\text { colmatées }\end{array}$ & Grottes & $\begin{array}{c}\text { Pertes } \\
\text { de ruisseaux }\end{array}$ & $\begin{array}{c}\text { Gouftres } \\
\text { Nombre nj }\end{array}$ \\
\hline a moyen & 138 & 202 & 88 & 36 & 34 & 9 \\
\hline$r=0$ & 0,795 & 1,164 & 0,507 & 0,207 & 0,196 & 0,052 \\
$r=1$ & 0,451 & 0,312 & 0,602 & 0,813 & 0,822 & 0,949 \\
$r=2$ & 0,359 & 0,363 & 0,305 & 0,168 & 0,161 & 0,049 \\
$r=3$ & 0,143 & 0,211 & 0,077 & 0,017 & 0,016 & 0,0013 \\
$r=4$ & 0,038 & 0,082 & 0,013 & 0,0012 & 0,0009 & $2,20 \mathrm{E}-05$ \\
$r=5$ & 0,0012 & 0,024 & 0,0017 & $6,20 \mathrm{E}-05$ & $4,00 \mathrm{E}-05$ & $2,90 \mathrm{E}-07$ \\
$r=6$ & 0,00016 & 0,0056 & 0,00017 & $2,60 \mathrm{E}-06$ & $2,00 \mathrm{E}-06$ & $3,00 \mathrm{E}-09$ \\
\hline Cumul $\mathrm{Pr}$ & 0,9998 & 0,9986 & 0,000014 & $8,90 \mathrm{E}-08$ & $6,50 \mathrm{E}-08$ & $2,60 \mathrm{E}-11$ \\
\hline
\end{tabular}

TAaIEAU III Répartitions espérées des événements.

\begin{tabular}{|c|c|c|c|c|c|c|}
\hline Évênement & Sources & $\begin{array}{l}\text { Dolines } \\
\text { actives }\end{array}$ & $\begin{array}{l}\text { Dolines } \\
\text { colmatees }\end{array}$ & Grottes & $\begin{array}{c}\text { Pertes } \\
\text { de ruisseaux }\end{array}$ & Gouffres \\
\hline a moyen & $\mathrm{a} 1=0,795$ & $a 5=1,164$ & $\mathrm{a} 3=0,507$ & $a 2=7,452$ & $a 6=6,664$ & $a 4=0,052$ \\
\hline $\begin{array}{l}r=0 \\
r=1 \\
r=2 \\
r=3 \\
r=4 \\
r=5 \\
r=6\end{array}$ & $\begin{array}{l}62 \\
50 \\
20 \\
5 \\
1 \\
0 \\
0\end{array}$ & $\begin{array}{l}63 \\
73 \\
43 \\
17 \\
5 \\
1 \\
0\end{array}$ & $\begin{array}{l}53 \\
27 \\
7 \\
1 \\
0 \\
0 \\
0\end{array}$ & $\begin{array}{c}29 \\
6 \\
1 \\
0 \\
0 \\
0 \\
0\end{array}$ & $\begin{array}{l}28 \\
5 \\
1 \\
0 \\
0 \\
0 \\
0\end{array}$ & $\begin{array}{l}9 \\
0 \\
0 \\
0 \\
0 \\
0 \\
0\end{array}$ \\
\hline Nombre nj & 138 & 202 & 88 & 36 & 34 & 9 \\
\hline
\end{tabular}

Expected distributed events. 
TABLEAU IV Répartitions espérées (Esp.) et observées (Obs.) des événements avec calcul du test du CHI². Remarque: La variable discrète $r$ a été limitée à 5 unités et la valeur du $\mathrm{CHI}^{2}$ est ici soulignée. Expected distribution (Esp.) and observed distribution (Obs.) of events with test estimation of CHF? Remark: The discrete variable $r$ is limited to 5 units and the $\mathrm{CH}^{2}$ value is underlined.

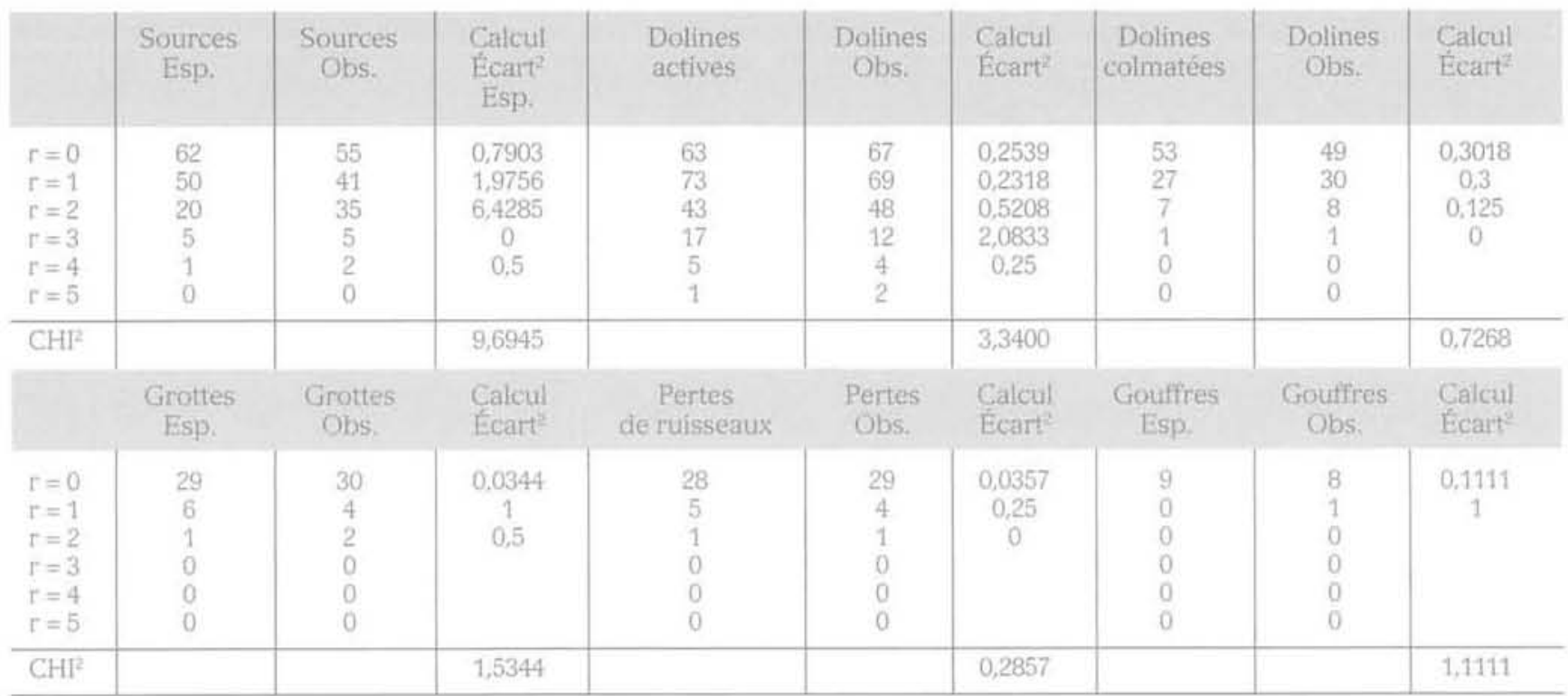

\section{3}

\section{Les critères physiques utiles}

\section{Objets recensés}

Sur le terrain puis sur les photographies aériennes toutes les formes exo-karstiques sont cartographiées: sources, pertes, dolines, grottes, failles. Deux catégories de dolines sont distinguées : les formes colmatées recouvertes par des dépóts résiduels argileux et ne montrant aucune trace d'activité actuelle et les formes actives. Sont considérées comme actives les dolines dans lesquelles les arbres sont inclinés ou courbés, ce qui traduit une mobilité récente du sol, ainsi que celles dans lesquelles la suffosion évacue des formations meubles de surface. De telles dolines montrent des effondrements actuels permettant le drainage des eaux vers la profondeur.

\section{2}

\section{Représentation cartographique}

La carte d'indices géomorphologiques distingue les zones suivantes:

-les alluvions anciennes et récentes. Ce paramétrage s'appuie sur une stabilité géotechnique apparente qui peut évoluer en cas d'inondation. L'existence d'alluvions actuellement stables ne préjuge pas de leur soutirage potentiel à l'avenir si une reprise des flux dans le karst sous-jacent réactive la suffosion. Les alluvions cartographiées, si elles sont anciennes, montrent une longue absence d'érosion. Si elles sont récentes, elles risquent de constituer le niveau de base local des écou- lements d'eau donc ont peu de chance de surmonter des écoulements actifs dans le karst et donc de se transformer à terme en un milieu à risque ;

-les zones calcaires très fracturées :

- les calcaires recouverts par des argiles à chailles ou par un recouvrement argileux ;

- la karstification située généralement sous une formation marneuse masquant des cavités profondes ;

- des dolines colmatées sur calcaires :

- des dolines fonctionnelles (avec un processus d'érosion visible) et des réseaux hydrologiques actifs sur calcaires.

La description des zones susceptibles de quider l'évaluation du risque de dégradation de la stabilité géotechnique au cours du temps passe par la définition d'un indice géomorphologique (Fig. 1) répondant à l'échelle suivante :

-indice 1 : zone alluvionnaire récente ou ancienne; -indice 2 : cavités reconnues sous un recouvrement marneux pouvant être continu :

-indice 3 : dolines colmatées stables;

- indice 4 : calcaire nu très fracturé ;

- indice 5 : doline active ou perte à écoulement temporaire :

-indice 6: contact linéaire par faille entre calcaires et marnes induisant des écoulements d'eau.

Des jeux de carte sont élaborés à l'échelle du $1 / 25000$ avec les relevés suivants :

- de zonation géomorphologique (Fig. 2a) ;

- de zonation indiquant la taille (diamètre) des cavités reconnues (Fig. 2b) ;

- de zonation indiquant la profondeur (ou hauteur) des cavités reconnues (Fig. 2c);

- de superposition des indices géomorphologiques, de taille et de profondeur aboutissant à une carte synthétique (Fig. 2d). 


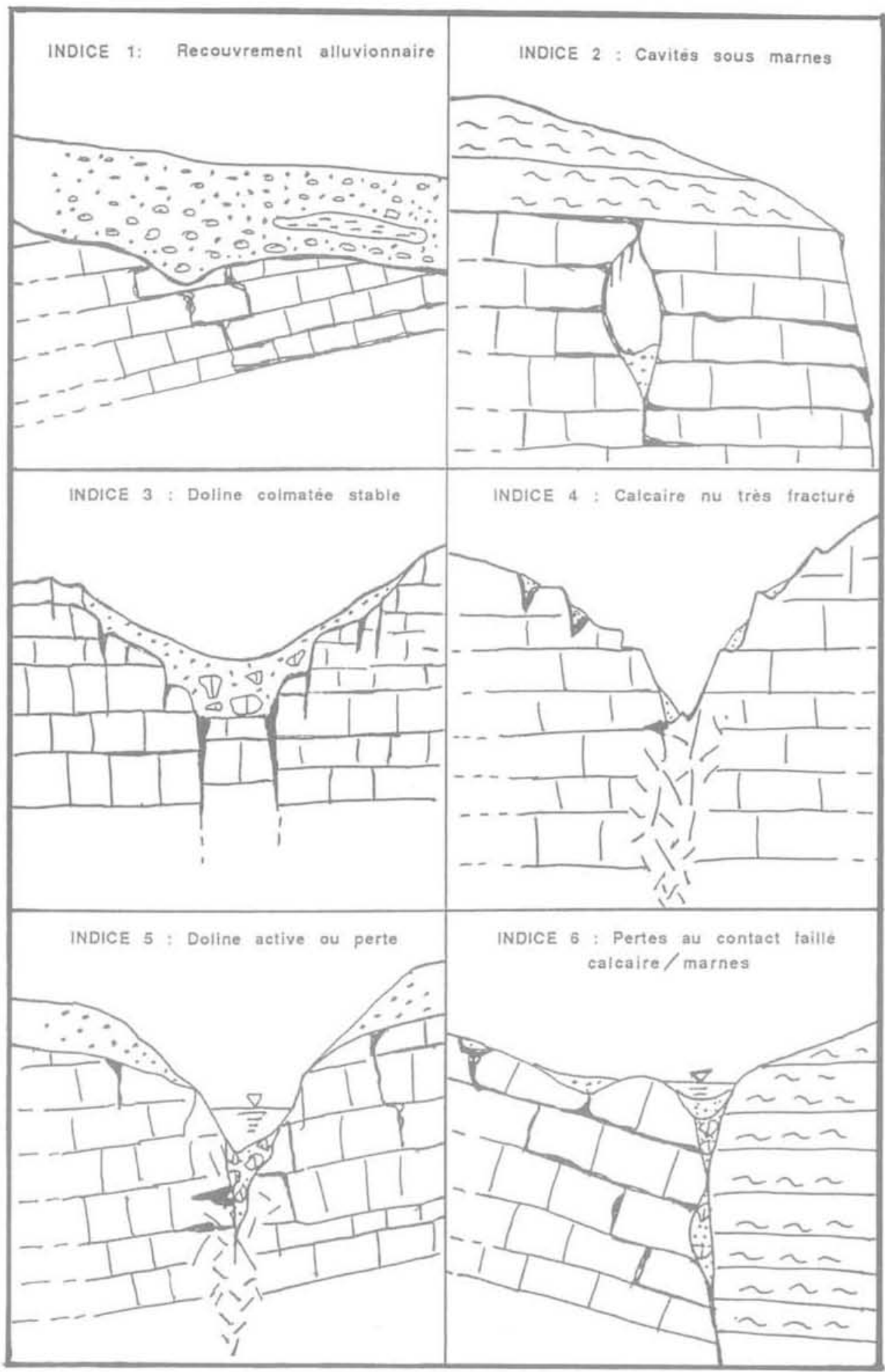

Indices géomorphologiques utilisés dans la cartographie des aléas karstiques des formations calcaires. Geomorphological index used in the cartography of karstic risk of limestones formations (East part of France).

Ces trois cartographies successives précisent la variation du diamètre, de la profondeur et du risque karstique,

La carte d'indice de taille (Fig. 2b) permet de définir la présence ou l'absence de cavités visibles en sub-surface et, dans le cas d'une présence, différentes tailles possibles (0 si pas de cavité, 1 pour un diamètre com- pris entre 0 et $5 \mathrm{~m}, 2$ pour un diamètre moyen de 5 à $25 \mathrm{~m}, 3$ pour un diamètre supérieur à $25 \mathrm{~m}$ ).

La carte d'indice de profondeur (Fig. 2c) définit la profondeur supposée de la cavité sous la surface du sol ( 1 à très grande profondeur, 2 pour 10 à $30 \mathrm{~m}, 3$ de 0 à $10 \mathrm{~m}$ en général visible).

La carte synthétique de vulnérabilité karstique 105 

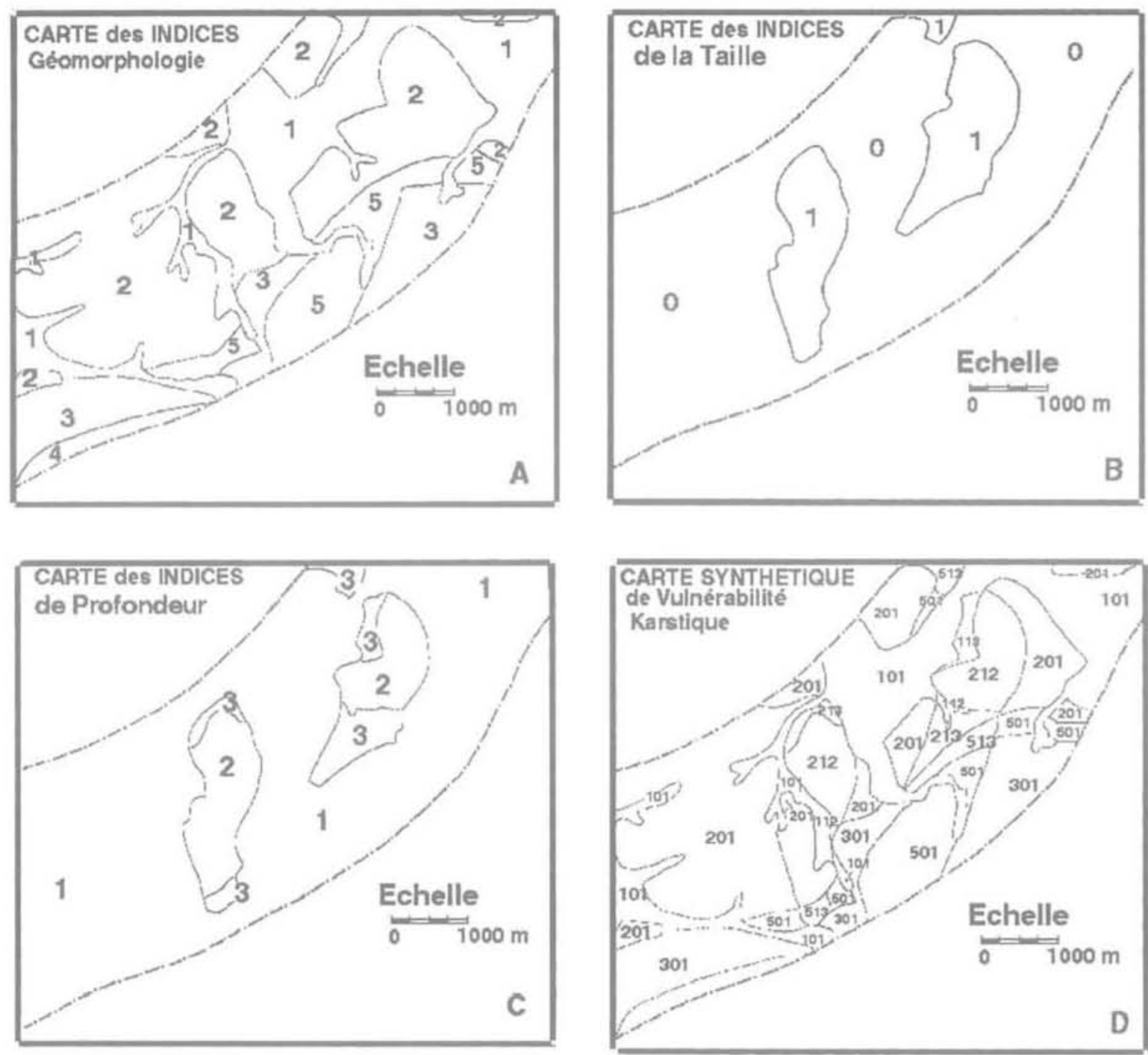

FI. 2 Exemple de carte de synthèse basée sur une zonéographie à 3 chiffres dans les formations calcaires (région Est de la France).

Example of synthetic map with zoneographical boundaries classified with 3 numbers across the limestones formations of Besancon country.

Carte A: indices géomorphologiques.

Map A: geomorphological index.

Carte B : taille des dolines.

(Légende: 0 : si pas de cavité; 1 : diamétre entre 0 et $5 \mathrm{~m} ; 2$ : diamétre moyen de 5 à $25 \mathrm{~m} ; 3$ : diamètre supérieur à $25 \mathrm{~m}$.) Map B: size of dolines.

(Legend: 0 : no cavity; 1 : diameter between 0 and $5 \mathrm{~m} ; 2$ : diameter between 5 and $25 \mathrm{~m} ; 3$ : diameter upper than $25 \mathrm{~m}$.) Carte C: profondeur des dolines.

(Légende : 1: faible probabilité de profondeur; 2: pour 10 à $30 \mathrm{~m} ; 3$ : de 0 à $10 \mathrm{~m}$ en général visible.)

Map C: deepth of dolines.

(Legend: 1: probable low possibility; 2 : between 10 and $30 \mathrm{~m}$; 3 ; visible between 0 and $10 \mathrm{~m}$.)

Carte $\mathrm{D}$ : synthèse générale de la carte des risques.

(Légende : chiffre 100 à 631 avec de gauche à droite; 1 : gèomorphologie; 2 : taille; 3 ; profondeur.)

Map D: general synthesis of the risk map.

(Legend: number 100 to 631 with from left to right : 1 : geomorphology: 2 : size; 3 : deepth.)

(Fig. 2d) regroupe les informations des 3 cartes indicées précédentes (géomorphologie, taille et profondeur). Cette carte permet de repérer rapidement les zones sensibles en juxtaposant les trois indices respectifs à la géomorphologie, à la taille et à la profondeur. En effet plus l'indice est élevé plus la zone est délicate et présente des aléas potentiels d'instabilité. Les zones les moins problématiques sont notées 101 alors que les plus dangereuses 633 . Cette carte synthétique reste très 10 descriptive d'où la nécessité de lui affecter un para- mètre “ aléa karstique » qui apporte un poids aléatoire au travers de l'application de la loi de Poisson.

\section{3}

\section{Définition de l'aléa karstique}

L'aléa karstique correspond à la fois à la nature de la discontinuité, à sa localisation et à sa fréquence d'appa- 
rition qui sont définies par un indice karstique $I_{k}$ unique qui nécessite l'évaluation successive :

- de la valeur de la superficie totale du fuseau $\left(S_{t t}\right)$ évaluée pour chaque planche cartographique ;

- du coefficient de risque zonal $\mathrm{R}$ de la planche obtenu par l'addition de termes élémentaires, ainsi

$$
R=S_{j} \cdot a_{j}+\ldots+S_{m} \cdot a_{m}
$$

avec $S$ la superficie de la zone indicée $j$ (1 à 6 pour l'indice géomorphologique), a le paramètre de la loi de Poisson (1) qui tiendra lieu de terme pondérateur allant d'un minimum de 0,052 pour a4 à un maximum de 7,452 pour a2 (Tableau III) de la zone indicée j calculé précédemment et $m$ le nombre maximal de zones. A titre d'exemple, pour la feuille de Pesmes ouest (PEO III) : $R=S_{1} a_{1}+S_{2} a_{2}+S_{5} a_{5}=116,84$;

- la répartition de la surface du fuseau en fonction des six indices géomorphologiques conduisant à la définition d'un coefficient maximum de risque $R_{\text {max }}=S_{\text {if }}\left(a_{1}+\ldots\right.$ $+a_{\text {J }}$. A titre d'exemple pour la feuille de Pesmes ouest (PEO III) : $R_{\max }=S_{t *}{ }^{*} 16,634=382,58$;

- du rapport $R / R_{\text {max }}$ nous donnant ainsi l'indice de karstification $I_{k}$ representant l'aléa karstique potentiel. A titre d'exemple pour la feuille de Pesmes ouest (PEO III) $: I_{\mathrm{k}}=R / R_{\max }=30,54 \%$.

On peut ainsi constater (Tableau V) que le risque zonal $R$ de la nouvelle méthode oscille entre les valeurs de 2,63 à 210,27 (ancienne méthode de 4,7 à 115,25 selon Mania et al., 1998), et que l'indice de karstification

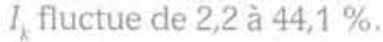

Cette nouvelle méthode d'estimation de l'aléa karstique qui prend en compte les paramètres de probabilité dilate la gamme de précision de l'échelle générale. Ainsi ce type d'information sera précieux pour les géotechniciens qui peuvent envisager des investigations géologiques et géophysiques renforcées sur les secteurs à fort aléa karstique.

On peut définir ensuite une règle simple pour une zonation cartographique en plaçant les secteurs dans des zones à très faible risque $($ si $0<\mathrm{R} 1<50)$ pour 13 planches, à risque moyen $(50<\mathrm{R} 2<100)$ pour 9 planches, à grand risque $(100<R 3<150)$ pour 6 planches et à haut risque (R4 > 150) pour une seule planche. Le choix du nombre des zones est ici adapté aux types d'intervention sur le génie civil (drainage, création de bassins de rétention, comblement de dolines, injection de cavités) et dans notre cas quatre zones semblent raisonnables.

\section{Incidences en termes de génie civil}

Les zones à très faible risque R1 laissent supposer qu'aucune précaution gêotechnique n'est utile pour le passage du tracé ferroviaire. Il ne faut pas cependant exclure certaines surprises comme dans le cas de la

TABLEAUV Estimation des coefficients de risque $R$ et des indices de karstification $I_{k}$. Risk coefficients $(R)$ and index of karstification $(I)$.

\begin{tabular}{|c|c|c|c|c|c|c|c|c|c|c|}
\hline \multirow{2}{*}{ Planche $n^{n}$} & \multirow[b]{2}{*}{$\begin{array}{c}\text { Distances } \\
\text { en km }\end{array}$} & \multirow{2}{*}{$\begin{array}{l}\text { Superficie } \\
\text { en } \mathrm{km}^{2}\end{array}$} & \multicolumn{6}{|c|}{ Superficie zonale par indice géomorphologique } & \multirow[b]{2}{*}{$\begin{array}{c}\text { Coeff: } \\
\text { R }\end{array}$} & \multirow[b]{2}{*}{$\begin{array}{c}\text { Indice I } \\
\text { en } \%\end{array}$} \\
\hline & & & Indice 1 & Indice 2 & Indice 3 & Indice 4 & Indice 5 & Indice 6 & & \\
\hline $\begin{array}{l}\text { PEO III } \\
\text { PEO IV } \\
\text { PEE I } \\
\text { PEE III } \\
\text { PEE II } \\
\text { PEE IV } \\
\text { GY III } \\
\text { GY II + IV } \\
\text { BEO I } \\
\text { BEO II } \\
\text { BEE I + II } \\
\text { VEE I + II } \\
\text { BAO I } \\
\text { BAO III } \\
\text { BAO II } \\
\text { BAE I } \\
\text { BAE II } \\
\text { LUO III } \\
\text { LUO IV } \\
\text { LUE III } \\
\text { LUE IV } \\
\text { BFO III } \\
\text { FID I } \\
\text { FLD II } \\
\text { FLD III } \\
\text { FLD IV } \\
\text { MAR III } \\
\text { MAR IV + DI. } \\
\text { AUX I }\end{array}$ & $\begin{array}{c}7 \\
7 \\
2 \\
6 \\
7 \\
2,5 \\
3 \\
12,5 \\
7 \\
7 \\
8 \\
4,5 \\
6 \\
1 \\
8 \\
7 \\
3,5 \\
7 \\
7 \\
7 \\
7 \\
1,5 \\
2,12 \\
5,8 \\
5,12 \\
7,25 \\
12,77 \\
5,3 \\
6,62\end{array}$ & $\begin{array}{c}23 \\
24 \\
3 \\
20 \\
14 \\
10 \\
6,2 \\
32,5 \\
22 \\
21 \\
23 \\
14 \\
18,1 \\
7,7 \\
24,8 \\
20 \\
3,5 \\
22,5 \\
22,5 \\
21 \\
23 \\
4,75 \\
6,02 \\
18,18 \\
13,78 \\
29,23 \\
28,91 \\
15,5 \\
16,96\end{array}$ & $\begin{array}{c}8,1 \\
2,5 \\
1,4 \\
1,1 \\
7,3 \\
0,5 \\
1 \\
3 \\
4,2 \\
4,8 \\
5,5 \\
3,75 \\
3,1 \\
1,2 \\
1,4 \\
5 \\
0 \\
3,5 \\
6 \\
1,5 \\
2,5 \\
0,75 \\
0 \\
0 \\
0 \\
0,84 \\
0,39 \\
6,24 \\
16,96\end{array}$ & $\begin{array}{c}14,8 \\
18,9 \\
1,5 \\
11,4 \\
4,9 \\
0,45 \\
1,6 \\
6 \\
12,1 \\
14,2 \\
11,7 \\
10,25 \\
6 \\
6,5 \\
4,1 \\
11 \\
1,5 \\
16 \\
10 \\
5 \\
4,5 \\
0 \\
5,2 \\
13,61 \\
12,37 \\
27,19 \\
8.3 \\
0 \\
0\end{array}$ & $\begin{array}{c}0 \\
2,4 \\
0,1 \\
3,2 \\
1,3 \\
8,3 \\
1,2 \\
21,6 \\
2,7 \\
0 \\
2,7 \\
0 \\
8 \\
0 \\
16,6 \\
3,5 \\
1 \\
1 \\
0 \\
13,5 \\
11 \\
4 \\
0,01 \\
0,03 \\
0,06 \\
0,13 \\
0,08 \\
0,12 \\
0\end{array}$ & $\begin{array}{c}0 \\
0 \\
0 \\
0 \\
0 \\
0 \\
0 \\
0 \\
0 \\
0 \\
0,6 \\
0 \\
0 \\
0 \\
0 \\
0 \\
0 \\
0 \\
0 \\
0 \\
0 \\
0 \\
0 \\
0 \\
0 \\
0 \\
19,95 \\
9,14 \\
0\end{array}$ & $\begin{array}{c}0,1 \\
0,2 \\
0 \\
3,9 \\
0,5 \\
0,75 \\
2,2 \\
1,2 \\
3 \\
2 \\
2,7 \\
0 \\
1 \\
0 \\
2,7 \\
0,5 \\
1 \\
2 \\
6,5 \\
1 \\
4,5 \\
0 \\
0 \\
0,05 \\
0,08 \\
0,04 \\
0,19 \\
0 \\
0\end{array}$ & $\begin{array}{c}0 \\
0 \\
0 \\
0,4 \\
0 \\
0 \\
0,2 \\
0,7 \\
0 \\
0 \\
0 \\
0 \\
0 \\
0 \\
0 \\
0 \\
0 \\
0 \\
0 \\
0 \\
0,5 \\
0 \\
0,81 \\
4,49 \\
1,27 \\
1,03 \\
0 \\
0 \\
0\end{array}$ & $\begin{array}{c}116,85 \\
144,28 \\
12,35 \\
94,66 \\
43,56 \\
8,84 \\
17,23 \\
64,11 \\
98,37 \\
111,97 \\
96,11 \\
79,37 \\
52,4 \\
49,4 \\
43,23 \\
88,31 \\
12,85 \\
124,85 \\
86,86 \\
46,47 \\
49,67 \\
2,63 \\
44,16 \\
131,42 \\
100,77 \\
210,27 \\
63,47 \\
5,5 \\
13,49\end{array}$ & $\begin{array}{c}30,6 \\
36,2 \\
24,8 \\
28,5 \\
18,8 \\
5,4 \\
16,8 \\
11,9 \\
26,9 \\
32,1 \\
25,2 \\
34,1 \\
17,5 \\
38,6 \\
10,5 \\
26,6 \\
22,1 \\
33,4 \\
23,3 \\
13,4 \\
13 \\
3,4 \\
44,1 \\
43,5 \\
44 \\
43,3 \\
13,2 \\
2,2 \\
4,8\end{array}$ \\
\hline Total & 173,48 & 509,13 & 92,53 & 239,07 & 102,53 & 29,69 & 36,11 & 9.4 & & \\
\hline
\end{tabular}


planche MAR IV + DIJ (Tableau V) où la valeur faible de $R(=5,5)$ montre cependant l'existence de nombreuses sources dont le devenir doit être pris en considération pour éviter des inondations locales.

Les zones à risque moyen $\mathrm{R} 2$ pourraient entrainer un simple comblement de la doline.

Les zones à grand risque R3 pourraient amener à une réflexion sur une injection ou un comblement.

Les zones à haut risque $\mathrm{R} 4$ nécessiteraient systématiquement:

- un drainage amont des eaux superficielles évitant ainsi l'entrainement des particules;

- un traitement par comblement par des matériaux naturels et l'éventualité d'une injection par des matériaux de substitution des cavités souterraines.

Cette méthodologie doit être confortée par des reconnaissances géomécaniques et géophysiques qui devront confirmer ou infirmer nos premières estimations réalisées à partir d'observations externes qui dans certains cas pourraient ne pas faire apparaitre les réels désordres karstiques existant en profondeur.

\section{5}

\section{Impact sur les circulations d'eau}

Les observations effectuées sur le terrain conduisent à penser que des modifications probables des écoulements d'eau superficielle et d'eau souterraine dans les zones à haut risque pourront apparaître lors de la création d'infrastructures routières ou ferroviaires. On peut évoquer successivement:

- une diminution possible du débit d'étiage et une augmentation des débits de crue des sources si la géométrie des fractures est trop modifiée (par dé-colmatage des argiles) :

- un accroissement des débits de ruissellement (par effet de barrage des remblais et par comblement des cavités sous le linéaire).
On évitera de trop modifier l'état naturel des écoulements d'eau en veillant à :

- ne pas colmater les réseaux karstiques lors des terrassements :

- mettre en place des bassins de décantation le long des secteurs vulnérables :

- ménager les écoulements souterrains en compatibilité avec les données environnementales.

\section{6}

\section{Conclusion}

Une nouvelle méthode d'évaluation des risques liés à la dissolution de calcaires karstifiés est proposée à partir de la loi de Poisson. Nous avons développé les différentes étapes du calcul et le test de validité associé. Cette nouvelle méthode d'estimation du risque zonal $\mathrm{R}$ prend en compte les paramètres de probabilité Elle permet de dilater la gamme de précision de l'échelle génèrale donc la sensibilité de l'outil.

La combinaison entre l'outil statistique et l'observation des événements liés à la dissolution des calcaires permet d'améliorer la cartographie des risques en apportant un moyen efficace pour l'orientation des investigations géotechniques et d'environnement. Nous avons présenté une application sur 19 planches affectées à un tracé linéaire sur une distance de $173,5 \mathrm{~km}$ et $509 \mathrm{~km}^{2}$. Ainsi une zonation en degré de risque croissant (faible, moyen, grand et à haut risque) est proposée. Les secteurs où figurent le maximum de contraintes d'ordre géologique, induiront automatiquement des études complémentaires détaillées de type géophysique, sondages géotechniques et investigations géomécaniques.

\section{REMERCIEMENTS}

Je remercie le responsable de la mission Rhin-Rhòne de la SNCF et la Direction de l'équipement et de l'aménagement de la SNCF à Paris. Que les relecteurs scientifiques sojent remerciés pour les critiques constructives.

\section{$\overline{\text { Bibliographie }}$}

Doerfliger N., Zwalhen F., Meylan B., Tripet j.P. Wilberger A. - Vulnérabilité des captages en milieu karstique, Zurich. GWA $5 / 97,1997$, p. 295-302.

Fares A. Rollet M. Broquet P. - « Méthodologie de la cartographie des risques naturels liés aux mouvements de terrain (région de Taounate-Rif, Nord du Maroc) „. Revue francaise de géotechnique, 69, 4, 1994, p. 63-72
Mania J., Wacogne R., Delhomel A. "Délimitations des zones à aléas karstiques entre les vallées de la Saóne et la vallée de la Savoureuse (TGV RhinRhóne) m. Colloque Tectonique, karst et séismes de Han-sur-Lesse, Belgique, 1998, 4 p.

Sanejouand R. - La cartographie géotechnique en France. Laboratoire central des ponts et chaussées et Armines, 1972, $96 \mathrm{p}$.
Tripet J.P.. Doerfliger N., Zwalhen F. "Vulnerability mapping in karst areas and its uses in Switzerland $\pi$. Hydrogéologie, Orléans, BRGM, 3, 1997, p. 5157.

Vrba J., Zaporozec A. - Guidebook on mapping groundwater vulnerability. Hannover, International Association of Hydrology, 16, 1994, $131 \mathrm{p}$. 